\title{
Accuracy of periapical and panoramic radiography for detection of root morphologies: a comparative study
}

\section{Esma J. DOĞRAMACI(a) \\ Daniel de Almeida DECURCIO(b) Catherine Jiayuan CHEN $^{(a)}$ Carlos ESTRELA ${ }^{\text {(b) }}$ Giampiero ROSSI-FEDELE(a)}

(a) University of Adelaide, Adelaide Dental School, Adelaide, Australia.

(b) Universidade Federal de Goiás - UFG, Faculty of Dentistry, Department of Stomatological Sciences, Goiânia, Brazil.

Declaration of Interests: The authors certify that they have no commercial or associative interest that represents a conflict of interest in connection with the manuscript.

Corresponding Author:

Giampiero Rossi-Fedele

E-mail: giampiero.rossi-fedele@adelaide.edu.au

Submitted: June 30, 2020

Accepted for publication: December 9, 2020

Last revision: February 2, 2021

\begin{abstract}
Maxillary and mandibular incisors have increased risk for severe orthodontically induced inflammatory root resorption. A patient-related risk factor is aberrant root morphology. This study aimed to assess the frequency of detection of different root morphologies in anterior teeth using dental panoramic tomography (DPT) and long cone periapical radiographs (LCPAs). A retrospective cross-sectional design was used to assess a sample of 50 consecutive pre-treatment radiographic records of patients from a specialist orthodontic practice in Adelaide, Australia. A reference guide was developed that included three previously unreported morphologies: pipette and bent, bent and pointed, bent and blunt. Two trained and calibrated assessors examined each record against the inclusion criteria, then independently assessed each anterior tooth from DPTs and LCPAs to detect the type of root morphology present. Data were analysed using the chi-square statistical test. Radiographic records for 48 patients (48 DPTs and 161 LCPAs) were eligible, with 355 and 426 teeth on DPTs and LCPAs, respectively, included for assessment. Normal root morphology (119 teeth) was commonly observed in DPTs, while bent (154 teeth) was frequently observed using LCPAs. Mandibular incisors often had normal morphology in DPTs but bent in LCPAs. Bent was the most common morphology in maxillary lateral incisors using DPT and LCPAs, although maxillary centrals were mostly normal in DPTs but pointed in LCPAs. Differences using the two image acquisition methods were highly significant $(p<0.01)$. Aberrant root morphologies are more easily detected in anterior teeth using LCPAs compared to DPTs.
\end{abstract}

Keywords: Diagnostic Techniques and Procedures; Iatrogenic Disease; Root Resorption.

\section{Introduction}

Orthodontically induced inflammatory root resorption (OIIRR) is an undesirable iatrogenic outcome during the course of orthodontic treatment, characterised by a sterile and aggressive inflammatory resorptive process that could involve the outer cemental layers, the outer cemental and dentinal layers, or full resorption of all hard tissues at the apex of a root. ${ }^{1}$ The usual extent of root length loss following conventional fixed orthodontic treatment, determined radiographically, is less than $2.5 \mathrm{~mm}, 2,3,4$ 
which is considered clinically acceptable. However, severe resorption, defined as greater than $4 \mathrm{~mm}$ or a third of the original root length, ${ }^{5,6,7}$ can jeopardise the outcome of orthodontic treatment, ${ }^{7}$ with one of the possible outcomes being increased tooth mobility, on account of the reduction in intra-alveolar root length changing the crown:root ratio, leading to possible tooth loss. ${ }^{8}$ Restorative replacement with either removable or fixed prosthodontics, or a single tooth implant, present significant difficulties and limitations, ${ }^{9,10}$ which would be marked in the case of a mandibular incisor.

Tooth type and root morphology are recognised as important patient-related variables that can influence a patient's susceptibility to experiencing severe OIIRR. ${ }^{5,11}$ Maxillary lateral incisors most commonly experience severe OIIRR ${ }^{2,4,11}$ although other anterior teeth in the maxilla and mandible can also be affected. ${ }^{4,12}$ Teeth with abnormal root morphologies are an important risk factor, $3,4,6,11,12,13,14$ with those described as apically bent, ${ }^{4,5,14}$ blunt, ${ }^{5,13}$ eroded ${ }^{14}$ narrow, ${ }^{4,12}$ pipette shaped, ${ }^{4,5,14}$ and pointed ${ }^{4,14}$ being most susceptible. Therefore, a risk assessment for the development of severe OIIRR in a prospective orthodontic patient should include determination of the presence of any abnormal root morphology in anterior teeth. This would ideally be performed radiographically after an initial clinical examination, as part of the diagnosis and treatment planning stage.

Two-dimensional extra- and intra-oral radiography techniques are commonly used in orthodontics. ${ }^{15,16}$ Most of the existing literature into OIIRR uses dental panoramic tomography (DPT) for the assessment of incisors. This technique produces a single image that includes both the maxillary and mandibular dentitions and adjacent structures. ${ }^{16}$ However, one of the disadvantages of DPTs is the superimposition of the artefactual shadow of the cervical vertebrae in the region of the incisors. ${ }^{17}$ Additionally, errors in patient positioning or aberrant tooth positions owing to skeletal or dental sagittal discrepancies may produce images where teeth are either out of focus, or may not have been captured at all, since they were not within the focal trough. ${ }^{16,17}$ Previous work comparing the accuracy of diagnosis of root morphologies of maxillary and mandibular teeth using both DPT and long-cone periapical radiography (LCPA) showed root abnormalities were more difficult to detect on DPTs compared with LCPAs, with measurements of root lengths and resorption, particularly involving mandibular incisors, often being compromised because of distortion. ${ }^{18}$ However, no distinction was made between the assessed teeth, which included anterior teeth and premolars. This is of clinical significance as one of the factors influencing OIIRR severity is the type of tooth. When comparing maxillary lateral and central incisors, severe OIIRR following orthodontic treatment is more prevalent in the former. ${ }^{11}$

To the best of our knowledge, no studies have compared the accuracy of DPT versus LCPAs for the assessment of apical root morphology of anterior teeth, including mandibular incisors. Therefore, the aim of this study was to assess the frequency of detection of different root morphologies in maxillary and mandibular anterior teeth using DPTs and LCPAs. The null hypothesis tested was there is no difference in detection of root abnormalities in anterior teeth on DPTs and LCPAs.

\section{Methodology}

\section{Radiographic examination}

This retrospective cross-sectional study received ethical approval from the Human Research Ethics Committee of the University of Adelaide (H-2018-120). The study examined de-identified radiographic records of 50 consecutive patients, who comprised a sample of convenience, from a specialist orthodontic practice in Adelaide, Australia. Periapical radiographs were obtained with an intra-oral x-ray machine, whereas DPTs were performed with a Siemens OP3 machine (Siemens AG, Postfach, Nuernberg, Germany).

\section{Inclusion and exclusion criteria}

The inclusion criteria stipulated that each set of radiographic records must include a pre-treatment DPT and LCPAs of maxillary and mandibular anterior teeth that were of excellent or diagnostically acceptable quality, ${ }^{19}$ which was assessed by the authors prior to commencing the study. Only permanent anterior 
teeth (incisors and canines) with fully developed roots were eligible for analysis. Teeth with incompletely developed roots, unclear root morphology because of superimposition or artefactual shadows, teeth with radiographic evidence of endodontic treatment, or radiographic images rated as unacceptable i.e. errors of exposure, positioning or processing, were excluded.

\section{Assessment of root morphologies}

A scoping review to assess the types of roots evident on the radiographs was conducted a priori, which highlighted several teeth with roots displaying dual morphologies that were classified as pipette and bent, bent and pointed and bent and blunt. As these morphologies were not available in previously published guides, $, 3,5,20$ they were included in a guide specifically developed for this study (Figure 1). Prior to performing the analyses, two trained and calibrated assessors, a specialist (DAD) and general dental practitioner (CC), participated in intra- and interexaminer reliability assessments using a randomly selected sample of $10 \%$ of included radiographs, with a two-week interval between observations.

The assessors examined the radiographs, independently and in duplicate, using a fluorescent light box in a purpose-built dark room with black-out curtains, without the use of any additional magnification aids. In the case of multiple LCPAs available in a radiographic record, each visible tooth was assessed only once (i.e. not double counted). The following variables were assessed for each radiograph and recorded on a data collection form: 1. type of radiograph: DPT; LCPA, 2. type of anterior teeth visible, 3. type of root morphology detected on each visible anterior tooth. The data were then synthesised, with any disagreements resolved through discussion until a decision was obtained by consensus, including assistance by the other authors as required.

\section{Statistical analysis}

Intra- and inter-examiner reliability were evaluated using paired t-tests and Cohen's kappa respectively. Data were analysed using the chi-square statistic to test the frequency of the different types of root morphologies detectable on DPT and LCPAs, the frequency of the different morphologies based on the type of imaging modality (i.e. DPT or LCPAs) and type of root morphology against the specific type of tooth. Statistical significance was set at $\mathrm{P}<0.05$. All analyses were performed using IBM SPSS Statistics, version 20 (IBM Corp, Armonk, USA).

\section{Results}

Forty-eight patient radiographic records were eligible for assessment; two records were incomplete

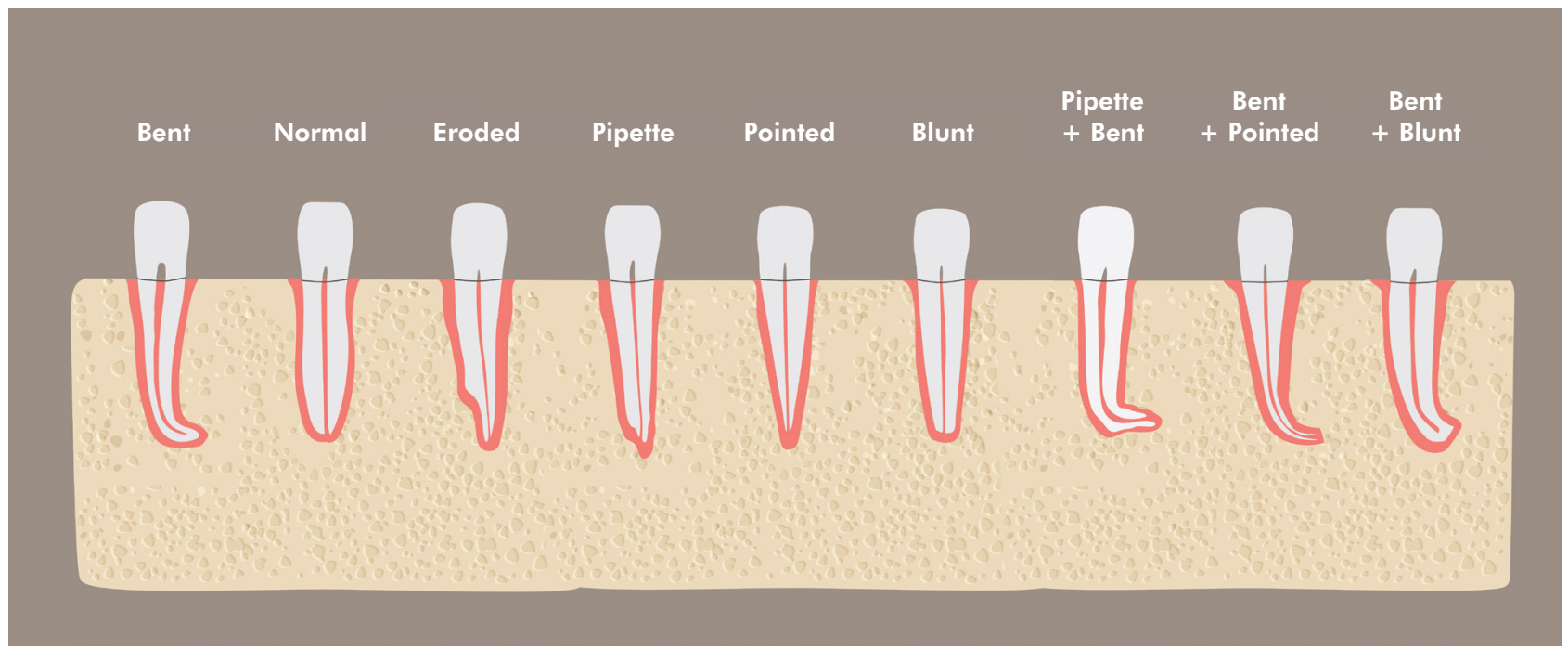

Figure 1. Root morphology guide. 
as they did not contain LCPAs and were therefore excluded. A total of 209 radiographs; 48 DPTs and 161 LCPAs, that had imaged 573 maxillary and mandibular anterior teeth were available for initial assessment. One patient was missing two maxillary lateral incisors while another patient was missing a single mandibular lateral incisor. The principal reason for exclusion was unobservable roots; 168 on DPTs, 103 on LCPAs. This was followed by incomplete root development; 43 on DPTs, 37 on LCPAs. Finally, there were seven teeth with evidence of previous endodontic treatment and these were also excluded. This gave 355 teeth on DPTs and 426 teeth on LCPAs for further analysis. Results of intra-examiner agreement produced almost perfect agreement with scores of $90.4 \%$ and $86.8 \%$. Inter-examiner reliability was almost perfect agreement (0.87).

The most common root morphology detected on teeth using DPTs was normal (119 teeth) while bent morphology was most frequently found in teeth using LCPAs (154 teeth) (Table 1). Chi-square test analysis indicated significant differences when comparing the frequency of the different types of root morphologies detected using the two radiographic techniques $(p<0.01)$. On DPTs, maxillary and mandibular central incisors mostly had normal root morphology, as did mandibular lateral incisors and mandibular canines (Table 2). Maxillary lateral incisors largely had bent roots while maxillary canines mostly had pointed roots (Table 2). When assessing using LCPAs, the canine teeth were frequently found to have normal morphology (Table 3). Bent morphology was commonly detected in maxillary laterals and mandibular incisors (Table 3). Pointed morphology was often found in maxillary central incisors (Table 3 ). The chi-square test indicated highly significant differences when comparing type of root morphology against the specific type of tooth, using either DPTs or LCPAs $(p<0.00)$. Three novel root morphologies were detectable using both image acquisition methods. Over $10 \%$ of teeth were found to have a bent and pointed morphology when assessed using DPTs and LCPAs, with this morphology detectable on all types of anterior teeth (Figure 2a). A pipette and blunt morphology was only detectable on lateral incisors (Figure 2b), while a bent and blunt morphology was found on a single maxillary lateral incisor using a DPT (Figure 2c).

\section{Discussion}

To the best of our knowledge, this is the first study examining the frequency of detection of different root morphologies in mandibular anterior teeth using DPTs and LCPAs. The most common

Table 1. Results of imaging diagnostic tests (panoramic and periapical) for the presence of normal and aberrant root morphologies.

\begin{tabular}{|c|c|c|c|c|c|}
\hline \multirow{3}{*}{ Type of morphology detected } & \multicolumn{4}{|c|}{ Image acquisition method } & \multirow{3}{*}{$\mathrm{p}$-value } \\
\hline & \multicolumn{2}{|c|}{ Dental panoramic tomography } & \multicolumn{2}{|c|}{ Long cone periapical } & \\
\hline & $\mathrm{n}$ & $\%$ & $\mathrm{n}$ & $\%$ & \\
\hline Normal & 119 & 33.5 & 100 & 23.5 & 0.011 \\
\hline Bent & 99 & 27.9 & 154 & 36.2 & \\
\hline Pointed & 71 & 20.0 & 97 & 22.8 & \\
\hline Bent + Pointed & 39 & 11.0 & 56 & 13.1 & \\
\hline Blunt & 17 & 4.8 & 11 & 2.6 & \\
\hline Pipette + Blunt & 5 & 1.4 & 2 & 0.5 & \\
\hline Pipette & 4 & 1.1 & 6 & 1.4 & \\
\hline Bent + Blunt & 1 & 0.3 & 0 & 0.0 & \\
\hline Eroded & 0 & 0.0 & 0 & 0.0 & \\
\hline Total & 355 & 100 & 426 & 100 & \\
\hline
\end{tabular}

${ }^{*}$ Chi-square test. 
Table 2. Results of dental panoramic tomography radiographs for the presence of normal and aberrant root morphologies.

\begin{tabular}{|c|c|c|c|c|c|c|c|}
\hline \multirow{4}{*}{$\begin{array}{l}\text { Type of morphology } \\
\text { detected }\end{array}$} & \multicolumn{6}{|c|}{ Tooth type } & \multirow{4}{*}{$p$-value } \\
\hline & \multicolumn{3}{|c|}{ Maxillary } & \multicolumn{3}{|c|}{ Mandibular } & \\
\hline & $\begin{array}{c}\text { Canine } \\
(\mathrm{n}=54)\end{array}$ & $\begin{array}{l}\text { Lateral incisor } \\
\quad(\mathrm{n}=59)\end{array}$ & $\begin{array}{l}\text { Central incisor } \\
(\mathrm{n}=53)\end{array}$ & $\begin{array}{c}\text { Canine } \\
(\mathrm{n}=62)\end{array}$ & $\begin{array}{l}\text { Lateral incisor } \\
\quad(n=63)\end{array}$ & $\begin{array}{l}\text { Central incisor } \\
(\mathrm{n}=64)\end{array}$ & \\
\hline & $\%$ & $\%$ & $\%$ & $\%$ & $\%$ & $\%$ & \\
\hline Normal & 11.1 & 18.6 & 49.1 & 30.6 & 46.0 & 43.8 & \multirow{9}{*}{0.000} \\
\hline Bent & 27.8 & 54.2 & 11.3 & 21.0 & 25.4 & 26.6 & \\
\hline Pointed & 29.6 & 6.8 & 34.0 & 17.7 & 12.7 & 21.9 & \\
\hline Bent + Pointed & 16.7 & 16.9 & 5.7 & 11.3 & 11.1 & 4.7 & \\
\hline Blunt & 11.1 & & & 11.3 & 3.2 & 3.1 & \\
\hline Pipette + Blunt & & 1.7 & & 4.8 & 1.6 & & \\
\hline Pipette & 3.7 & & & 3.2 & & & \\
\hline Bent + Blunt & & 1.7 & & & & & \\
\hline Eroded & & & & & & & \\
\hline
\end{tabular}

Table 3. Results of long-cone periapical radiographs for the presence of normal and aberrant root morphologies.

\begin{tabular}{|c|c|c|c|c|c|c|c|}
\hline \multirow{4}{*}{$\begin{array}{l}\text { Type of morphology } \\
\text { detected }\end{array}$} & \multicolumn{7}{|c|}{ Tooth type } \\
\hline & \multicolumn{3}{|c|}{ Maxillary } & \multicolumn{3}{|c|}{ Mandibular } & \multirow{3}{*}{$p$-value* } \\
\hline & $\begin{array}{c}\text { Canine } \\
(\mathrm{n}=55)\end{array}$ & $\begin{array}{l}\text { Lateral incisor } \\
\quad(\mathrm{n}=87)\end{array}$ & $\begin{array}{c}\text { Central incisor } \\
(\mathrm{n}=83)\end{array}$ & $\begin{array}{c}\text { Canine } \\
(n=29)\end{array}$ & $\begin{array}{l}\text { Lateral incisor } \\
\qquad(\mathrm{n}=84)\end{array}$ & $\begin{array}{c}\text { Central incisor } \\
(\mathrm{n}=88)\end{array}$ & \\
\hline & $\%$ & $\%$ & $\%$ & $\%$ & $\%$ & $\%$ & \\
\hline Normal & 32.7 & 10.3 & 31.3 & 41.4 & 23.8 & 17.0 & 0.000 \\
\hline Bent & 18.2 & 52.9 & 16.9 & 34.5 & 42.9 & 43.2 & \\
\hline Pointed & 23.6 & 8.0 & 42.2 & 17.2 & 20.2 & 22.7 & \\
\hline Bent + Pointed & 20.0 & 25.3 & 2.4 & 3.4 & 8.3 & 14.8 & \\
\hline Blunt & 3.6 & 1.1 & 6.0 & & 3.6 & & \\
\hline Pipette + Blunt & & 2.3 & & & & & \\
\hline Pipette & 1.8 & & 1.2 & 3.4 & 1.2 & 2.3 & \\
\hline \multicolumn{8}{|l|}{ Bent + Blunt } \\
\hline Eroded & & & & & & & \\
\hline
\end{tabular}

morphology detectable on DPTs was normal while it was bent in LCPAs. Although mandibular central incisors were commonly observed as having a normal root morphology in DPTs, both the central and lateral mandibular incisors frequently had a bent morphology when assessed using LCPAs. This is a clinically important and previously unreported finding as a bent morphology is associated with severe OIIRR. Moreover, this implies that sole reliance on DPTs for assessing a patient's anterior teeth will result in more frequent detection of normal root morphologies, though the morphology is likely to be different when assessed using LCPAs. Owing to the significant differences in the detection of root abnormalities in anterior teeth on the DPTs and LCPAs, the research null hypothesis was therefore rejected.

The radiographic technique used may influence the prognostication of OIIRR. Root morphologies assessed from LCPAs as being blunt or pipette shaped prior 

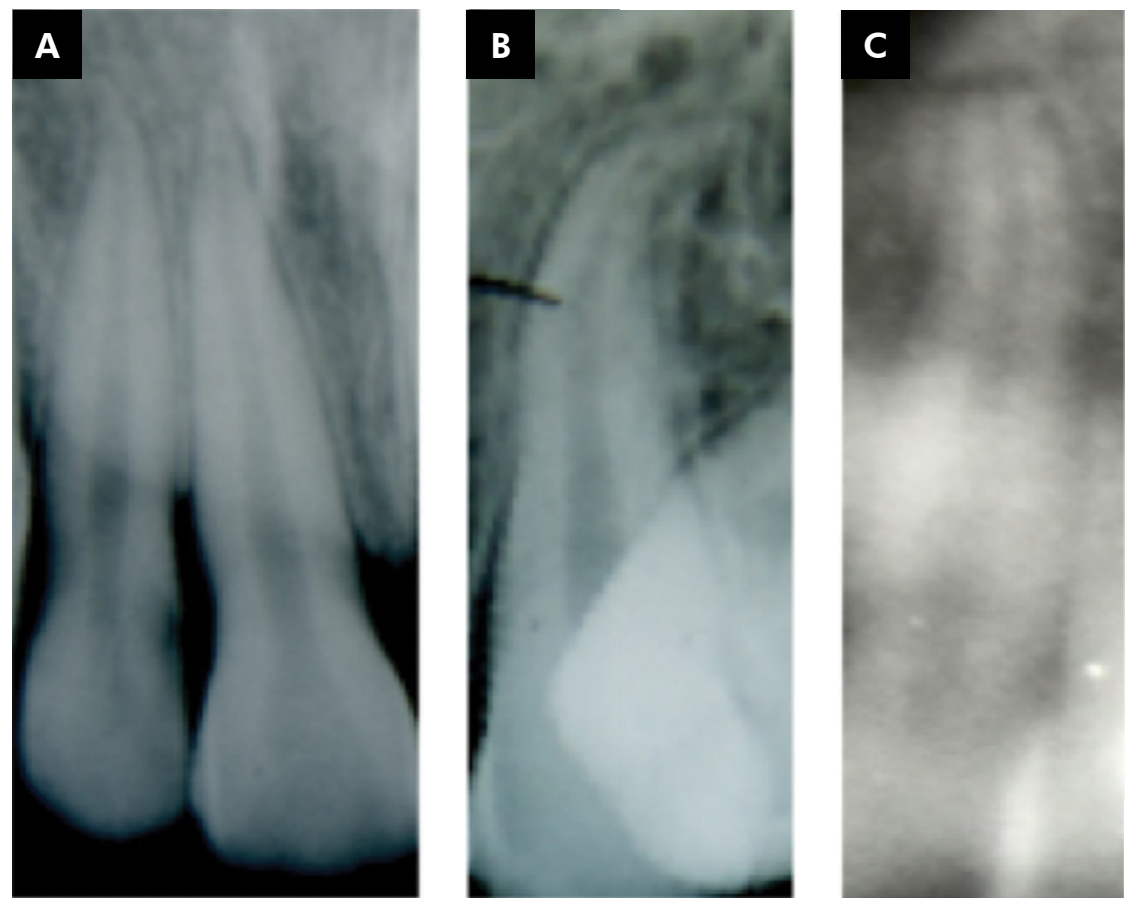

Figure 2. Examples of novel dual root morphologies: a) pipette and bent, b) bent and pointed, c) bent and blunt.

to orthodontic treatment have been found to have a significant association with severe OIIRR. ${ }^{5}$ Conversely, a study where pre-treatment assessment was carried out using DPTs suggested that roots with unusual morphology were at risk of moderate to severe OIIRR when compared to roots described as normal. ${ }^{11}$ This reiterates the importance of pre-operative assessment using intra-oral radiography in orthodontics.

The difference in types of morphologies observable in roots using DPTs is likely due to a number of factors that are inherent in the panoramic technique including the narrow focal trough, ${ }^{15,21}$ a decrease in image quality from the cervical spine and other anatomical superimpositions, ${ }^{21}$ and patient positioning errors including palatal air space. ${ }^{22}$ Furthermore, panoramic radiography consists of a curved image layer due to multiple centres of rotation of the x-ray beam. Thus, LCPA radiographs should be considered superior in depicting roots, particularly as they do not have the same limitations in image quality that affect panoramic radiography, including the radiographic image being acquired from the projection of a single, non-orbiting $\mathrm{x}$-ray beam. Intraoral radiographic techniques have been suggested to be more reliable, thus being more representative of the clinical presentation, than DPTs. ${ }^{20}$ Conversely, roots may be unobservable on LCPAs if the apical part of their root is not captured in the intra-oral film due to difficulties in positioning the imaging receptor, ${ }_{1}^{17}$ although the apex may be visible on DPTs; this is a problem encountered with canines, as they have the longest root length of any tooth.

The reference guide developed for this study may have improved assessor objectivity and reliability of diagnosis, however, some of the novel root morphologies proposed are yet to be clinically shown to be associated with severe OIIRR. Although subjectivity in interpretation and thus classification of deviant root morphologies may be asserted as being a limitation, assessment was undertaken only after examiner training and calibration was completed, with confirmation of achieving a high level of interand intra-examiner agreement before the study was commenced. An area of further research would be assessing whether roots with dual morphologies present an increased risk to severe OIIRR compared to roots having a single aberrant morphology.

Although it is not possible to directly compare our results with previous studies owing to the use 
of alternative classification systems, assessment of different groups of teeth, and radiographs being sourced from different settings and populations, a relatively higher detection rate of non-normal root morphology was evident in our study. Using DPTs, unusual morphology for maxillary incisors was previously reported to range between $35.5 \%$ and $40 \%,{ }^{11,20}$ whereas this study found a higher rate, $66.9 \%$. Another investigation using LCPAs found 36.5\% of maxillary incisors had unusual morphology, ${ }^{5}$ whereas our study found $79.4 \%$ using the same imaging technique. Previously reported percentages of deviant root morphologies detectable on DPTs range between $15-17 \%, 5,23$ and $22-24 \%$ using LCPAs; ${ }^{5,23}$ while the present study has detected a significantly higher rate of non-normal root morphologies among maxillary and mandibular anterior teeth using both DPTs and LCPAs.

A key strength of this study is the sourcing of radiographs from an orthodontic practice, vis-à-vis a dental hospital or an orthodontic department within a hospital, both being settings where the most complex patients that usually require multi-disciplinary care are seen..$^{24} \mathrm{~A}$ primary care setting, from where our radiographs were sourced, is where the majority of routine orthodontic treatment is provided to the general population, ${ }^{24}$ thus using radiographs from such setting best reflects the clinical norm and makes the results of this study readily translatable to the general practice setting. Although digital radiography is increasingly used in primary dental care settings, ${ }^{25}$ no studies have yet compared the assessment of apical root morphology using digital versus hard-copy radiographs.

The methods used for image acquisition reflects the differences observed in this study, with LCPAs, overall, allowing greater identification of aberrant root morphologies in anterior teeth compared to DPTs. The teeth that most commonly experience severe OIIRR are maxillary lateral incisors ${ }^{2,4,11}$ and this study found no difference between the image acquisition methods tested for this tooth type. No studies have indicated specific teeth for which taking LCPAs would be most advantageous, although available guidelines suggest the use of periapical radiographs to supplement panoramic radiographs in the incisal region where abnormalities are suspected.${ }^{15}$ Therefore, LCPAs focussed on the mandibular and maxillary incisors may be valuable for diagnosis of deviant root morphologies as they are susceptible tooth types for severe OIIRR and are often not clearly visible on DPTs. This was highlighted by the present investigation and has significant clinical significance, due to the difficulties in managing mandibular incisors in cases of reduced stability, or any space due to tooth loss, both of which might be OIIRRrelated outcomes. Conversely, further radiographic examination of canine teeth will be more difficult to justify, considering the limited risk of severe OIIRR for these teeth..$^{2,4,11}$ Moreover, radiographic exposures with LCPAs after a DPT has already been obtained needs justification, as patients should be exposed to the least amount of radiation whilst obtaining the maximum information for diagnosis. Since cone-beam computed tomography is commonly used in the specialist practice setting, ${ }^{26,27}$ comparative studies to assess the accuracy for the diagnosis of deviant root morphologies of this imaging technique should be considered in future.

Morphological characteristics of the root are patientrelated risk factors for severe OIIRR. Prior knowledge of root morphology can affect clinical-decision making in orthodontic treatment, for example, the type of appliance, amount of force, type of movement, and the duration of treatment,${ }^{4,28}$ particularly as both maxillary and mandibular incisors frequently show apical resorption when compared to other teeth. ${ }^{29}$ Patients that display root morphologies associated with severe OIIRR must be made aware of the risks at the outset of treatment, since modifications to treatment-related factors will not necessarily obviate the risk of severe OIIRR. This is an important point to discuss during the shared-decision making process when obtaining informed consent.

\section{Conclusions}

Aberrant root morphologies likely associated with increased risk for severe OIIRR are more easily detected in anterior maxillary and mandibular teeth of prospective orthodontic patients using LCPAs compared to DPTs. 


\section{References}

1. Brezniak N, Wasserstein A. Orthodontically induced inflammatory root resorption. Part 1: the basic science aspects. Angle Orthod. 2002;72(2):175-9. https://doi.org/10.1043/0003-3219(2002)07

2. Linge $L$, Linge $B O$. Patient characteristics and treatment variables associated with apical root resorption during orthodontic treatment. Am J Orthod Dentofacial Orthop. 1991 Jan;99(1):35-43. https://doi.org/10.1016/S0889-5406(05)81678-6

3. Mirabella AD, Årtun J. Risk factors for apical root resorption of maxillary anterior teeth in adult orthodontic patients. Am J Orthod Dentofacial Orthop. 1995 Jul;108(1):48-55. https://doi.org/10.1016/S0889-5406(95)70065-X

4. Sameshima GT, Sinclair PM. Predicting and preventing root resorption: part II. Treatment factors. Am J Orthod Dentofacial Orthop. 2001 May;119(5):511-5. https://doi.org/10.1067/mod.2001.113410

5. Levander E, Malmgren O. Evaluation of the risk of root resorption during orthodontic treatment: a study of upper incisors. Eur J Orthod. 1988 Feb;10(1):30-8. https://doi.org/10.1093/ejo/10.1.30

6. McNab S, Battistutta D, Taverne A, Symons AL. External apical root resorption of posterior teeth in asthmatics after orthodontic treatment. Am J Orthod Dentofacial Orthop. 1999 Nov;116(5):545-51. https://doi.org/10.1016/S0889-5406(99)70187-3

7. Levander E, Malmgren O. Long-term follow-up of maxillary incisors with severe apical root resorption. Eur J Orthod. Feb;22(1):85-92. https://doi.org/10.1093/ejo/22.1.85

8. Doğramaci EJ, Sherriff M, Rossi-Fedele G, McDonald F. Location and severity of root resorption related to impacted maxillary canines: a cone beam computed tomography (CBCT) evaluation. Aust Orthod J. 2015 May;31(1):49-58.

9. Al-Quran FA, Al-Ghalayini RF, Al-Zu'bi BN. Single-tooth replacement: factors affecting different prosthetic treatment modalities. BMC Oral Health. $2011 \mathrm{Dec} ; 11(1): 34$. Available from: https://doi.org/doi:10.1186/1472-6831-11-34 https://doi.org/10.1186/1472-6831-11-34

10. Rossi-Fedele G, Musu D, Cotti E, Doğramacı EJ. Root canal treatment versus single-tooth implant: a systematic review of Internet content. J Endod. 2016 Jun;42(6):846-53. https://doi.org/10.1016/i.joen.2016.02.011

11. Brin I, Tulloch JF, Koroluk L, Philips C. External apical root resorption in Class II malocclusion: a retrospective review of 1-versus 2-phase treatment. Am J Orthod Dentofacial Orthop. 2003 Aug;124(2):151-6. https://doi.org/10.1016/S0889-5406(03)00166-5

12. Sameshima GT, Sinclair PM. Characteristics of patients with severe root resorption. Orthod Craniofac Res. 2004 May;7(2):108-14. https://doi.org/10.1111/j.1601-6343.2004.00284.x

13. Newman WG. Possible etiologic factors in external root resorption. Am J Orthod. 1975 May;67(5):522-39. https://doi.org/10.1016/0002-9416(75)90298-5

14. Smale I, Årtun J, Behbehani F, Doppel D, Hof M, Kuijpers-Jagtman AM. Apical root resorption 6 months after initiation of fixed orthodontic appliance therapy. Am J Orthod Dentofacial Orthop. 2005 Jul;128(1):57-67. https://doi.org/10.1016/i.ajodo.2003.12.030

15. Isaacson KG, Thom AR, Atack NE, Horner K, Whaites E. Orthodontic radiographs: guidelines for the use of radiographs in clinical orthodontics. London: British Orthodontic Society; 2015 [cited 2020 Jun 21]. Available from: https://www.bos.org.uk/Portals/0/Public/ docs/General\%20Guidance/Orthodontic\%20Radiographs\%202016\%20-\%202.pdf

16. Abdelkarim A, Jerrold L. Clinical considerations and potential liability associated with the use of ionizing radiation in orthodontics. Am J Orthod Dentofacial Orthop. 2018 Jul;154(1):15-25. https://doi.org/10.1016/j.ajodo.2018.01.005

17. Whaites $E$, Drage N. Essentials of dental radiography and radiology. 5th ed. London: Elsevier Health Sciences; 2013.

18. Sameshima GT, Asgarifar KO. Assessment of root resorption and root shape: periapical vs panoramic films. Angle Orthod. 2001 Jun;71(3):185-9. https://doi.org/10.1043/0003-3219(2001)071<0185:AORRAR>2.0.CO;2

19. Royal College of Radiologists, National Radiological Protection Board. Guidelines on radiology standards for primary dental care. Chilton: National Radiological Protection Board; 1994.

20. Witcher TP, Brand S, Gwilliam JR, McDonald F. Assessment of the anterior maxilla in orthodontic patients using upper anterior occlusal radiographs and dental panoramic tomography: a comparison. Oral Surg Oral Med Oral Pathol Oral Radiol Endod. 2010 May; 109(5):765-74. https://doi.org/10.1016/i.tripleo.2009.10.016

21. Leach HA, Ireland AJ, Whaites EJ. Radiographic diagnosis of root resorption in relation to orthodontics. Br Dent J. 2001 Jan;190(1):16-22. https://doi.org/10.1038/sj.bdj.4800870

22. Granlund CM, Lith A, Molander B, Gröndahl K, Hansen K, Ekestubbe A. Frequency of errors and pathology in panoramic images of young orthodontics patients. 2012 Aug;34(4):452-7. https://doi.org/10.1093/ejo/cjr035.

23. Ahuja PD, Mhaske SP, Mishra G, Bhardwaj A, Dwivedi R, Mangalekar SB. Assessment of root resorption and root shape by periapical and panoramic radiographs: a comparative study. J Contemp Dent Pract. 2017 Jun;18(6):479-83. https://doi.org/10.5005/ip-journals-10024-2069

24. NHS England. Guide for commissioning orthodontics. London: NHS England; 2015 [cited 2020 June 21]. Available from: https://www.england.nhs.uk/commissioning/wp-content/uploads/sites/12/2015/09/guid-comms-orthodontics.pdf 
25. Russo JM, Russo JA, Guelmann M. Digital radiography: a survey of pediatric dentists. J Dent Child (Chic). 2006 Sep-Dec;73(3):132-5.

26. Doğramacı EJ, Rossi-Fedele G, McDonald F. Clinical importance of incidental findings reported on small-volume dental cone beam computed tomography scans focused on impacted maxillary canine teeth. Oral Surg Oral Med Oral Pathol Oral Radiol. 2014 Dec;118(6):e205-9. https://doi.org/10.1016/i.0000.2014.09.006

27. Borges C, Estrela C, Decurcio D, Pécora JD, Sousa-Neto M, Rossi-Fedele G. Cone-beam and micro-computed tomography for the assessment of root canal morphology: a systematic review. Braz Oral Res. 2020 Jun; 16;34:e056. https://doi: 10.1590/1807-3107bor-2020.vol34.0056

28. Han G, Huang S, Von den Hoff JW, Zeng X, Kuijpers-Jagtman AM. Root resorption after orthodontic intrusion and extrusion: an intraindividual study. Angle Orthod. 2005 Nov;75(6):912-8. https://doi.org/10.1043/0003-3219(2005)75[912:RRAOIA]2.0.CO;2

29. Apajalahti S, Peltola JS. Apical root resorption after orthodontic treatment: a retrospective study. Eur J Orthod. 2007 Aug;29(4):408-12. https://doi.org/10.1093/ejo/cjm016 